\title{
Pharmacokinetic Parameter Specimen Type
}

National Cancer Institute

\section{Source}

National Cancer Institute. Pharmacokinetic Parameter Specimen Type. NCI Thesaurus.

Code C87986.

The type of a material sample taken from a biological entity for pharmacokinetic parameter testing. 